EPJ Web of Conferences 116, 04007 (2016)

DOI: $10.1051 /$ epjconf/201611604007

(C) Owned by the authors, published by EDP Sciences, 2016

\title{
Neutrino fluxes from the Galactic plane and the ANTARES limit
}

\author{
Luigi Antonio Fusco ${ }^{1,2, a}$ on behalf of the ANTARES Collaboration \\ ${ }^{1}$ Dipartimento di Fisica e Astronomia dell'Università, Viale Berti-Pichat 6/2, 40127 Bologna, Italy \\ ${ }^{2}$ INFN - Sezione di Bologna, Viale Berti-Pichat 6/2, 40127 Bologna, Italy
}

\begin{abstract}
The existence of cosmic neutrinos has been reported by the IceCube Collaboration. Though this measurement is consistent with an isotropic neutrino flux, a sub-dominant galactic component coming from extended regions such as the Galactic Plane cannot be excluded. The ANTARES detector, located in the Mediterranean Sea, is currently the largest and longest operated under-water neutrino telescope; its effective area and good exposure to the Southern Sky allow to constrain an enhanced muon neutrino emission from extended sources such as the Galactic Plane. ANTARES data from 2007 to 2013 have been analysed and upper limits on the neutrino production from the central region of our galaxy have been set.
\end{abstract}

\section{Introduction}

The ANTARES detector [1] is currently the largest and longest operated under-water neutrino telescope. Its main scientific goal is to detect high energy neutrinos of cosmic origin. The IceCube Collaboration has reported the observation of a cosmic neutrino signal in the High Energy Starting Events (HESE) analysis. The purely atmospheric expectation is rejected at a level of $5.7 \sigma$ with three years of data [2]. This signal is compatible with an isotropic cosmic flux which is equally distributed in the three neutrino flavours [3]. However, some possible enhancement of the neutrino flux from the Southern hemisphere with respect to the Northern one has been underlined, for example in [4]. The low energy extension of the IceCube HESE analysis [5] shows a steepening of the energy spectrum of the cosmic signal producing more events at low energy. Even if the IceCube volume is much larger, the effective area of ANTARES in the region between 20 and $50 \mathrm{TeV}$ for the Southern Sky is similar [6]: some IceCube-like signal events could be observed by ANTARES too.

Because of the nature of the IceCube analysis, based on vetoing techniques to detect downwardgoing events, the signal is mainly distributed in the shower channel: the directional resolution for these events is poor. Since ANTARES is located in the Mediterranean Sea, the Southern sky is accessible to the detector via up-going tracks, for which sub-degree good angular resolution can be achieved [15]. A diffuse neutrino flux in the shower channel for IceCube might appear as an ensemble of individual point

\footnotetext{
a e-mail: lfusco@bo.infn.it
}

This is an Open Access article distributed under the terms of the Creative Commons Attribution License 4.0, which permits unrestricted use, distribution, and reproduction in any medium, provided the original work is properly cited. 
sources in ANTARES, or a region with an enhanced diffuse emission. The first possibility is addressed in [7], while the latter will be presented in this contribution.

\section{Neutrinos from the Milky Way}

Neutrinos can be produced close to galactic Cosmic Ray (CR) accelerators such as supernova remnants, when high energy protons or nuclei interact with the surrounding matter or photon field. Charged pions from those interactions decay into leptons (including neutrinos), while $\pi^{0}$, produced in equal amount at the interaction point, give $\gamma$-rays: the expected all-flavour neutrino flux from these interactions is equal to the $\gamma$ one. The neutrino energy spectrum directly follows that of CRs at the acceleration site $-\mathrm{E}^{-\Gamma}$ with $\Gamma=2.0$ for Fermi acceleration scenarios [8] - since the decay usually takes place before the primary particle can interact.

A contribution to a cosmic neutrino flux from the Milky Way is expected from interactions of CRs propagating in the inner region of our Galaxy. The neutrino spectrum should have the same spectral behaviour as propagating CRs, which is softer than that at sources. Data from the Fermi/LAT detector provide the best observation of this diffuse $\gamma$ flux in the Galactic Plane [9], though no observation of the neutrino counterpart is available. Assuming that a certain fraction of the observed diffuse $\gamma$ flux in the central region of the Galaxy originates from hadronic mechanisms, the neutrino yield from CR propagation can be calculated. Different predictions are available such in [10-12]. Each of these provides a different description of the expected neutrino flux, with an overall normalisation that can vary by one order of magnitude. A rather hard spectrum is expected, described, at least in part of the energy range, by a power law with spectral index $\Gamma \sim 2.4 \div 2.5$.

\section{The IceCube signal}

The one-flavour IceCube cosmic neutrino signal was initially [2] fitted by a power law spectrum with spectral index $\Gamma$ equal to 2 :

$$
E^{2} \frac{d \Phi}{d E}=(0.95 \pm 0.3) \times 10^{-8} \mathrm{GeV} \mathrm{s}^{-1} \mathrm{sr}^{-1} \mathrm{~cm}^{-2}
$$

while also an $\mathrm{E}^{-2.3}$ spectrum was reported to be compatible with the observed signal, mainly because of the absence of events above $2 \mathrm{PeV}$ and the lack of an enhanced neutrino detection in the region of the Glashow resonance $(\sim 6 \mathrm{PeV})$. The further extension of the IceCube data set and refinements in the analysis for the observation of lower energy neutrinos resulted in a steepening of the energy spectrum; in [5] the best fit is:

$$
\frac{d \Phi}{d E}=\left(2.06_{-0.3}^{+0.4}\right) \times 10^{-18}\left(\frac{E}{100 \mathrm{TeV}}\right)^{-2.46 \pm 0.12} \mathrm{GeV}^{-1} \mathrm{~s}^{-1} \mathrm{sr}^{-1} \mathrm{~cm}^{-2}
$$

for $25 \mathrm{TeV}<\mathrm{E}_{v}<1.4 \mathrm{PeV}$, rejecting the $E^{-2}$ hypothesis with a significance of $3 \sigma$. This flux is observed as an all sky flux by IceCube. This can be related to the bias in efficiency of the analysis to shower events, for which IceCube has a rather bad angular resolution (10 to 20 degrees). An excess of events close to the galactic plane is reported for example in [13]. This accumulation could point towards a diffuse emission region of cosmic neutrinos. In addition, as the Southern sky contains most of the Galactic Plane, the soft spectrum derived from IceCube measurement and such a possible enhancement of the neutrino flux in the Southern hemisphere hint towards a possible neutrino emission from the Galactic Plane. 


\section{Data analysis}

Assuming that part of the IceCube HESE signal comes from a region of the sky with angular size $\Omega \sim 0.1-0.2 \mathrm{sr}$, the corresponding neutrino flux can be computed from the IceCube effective area [4]. Such a signal, coming from an enhanced neutrino emission scenario can be searched for in ANTARES comparing the event rate from this area of the sky to what is observed where no signal is expected. A rectangular region having galactic coordinates $|1|<40^{\circ}$ and $|\mathrm{b}|<3^{\circ}$, enclosing the central part of the Fermi/LAT diffuse galactic plane flux, is chosen to represent the Galactic Plane area. Upward-going neutrino candidates collected by the ANTARES neutrino telescope from 2007 to 2013 are considered for this analysis.

\subsection{Atmospheric background}

The main background in neutrino telescopes is given by downward-going atmospheric muons reaching the detector. Since only neutrinos can traverse the Earth, neutrino telescopes look at upward-going events to reject this background. Wrongly reconstructed atmospheric muons, mimicking neutrino induced events, can be rejected by a selection on the track quality parameter $\Lambda$ and the estimate for the angular error $\beta$ [15].

Atmospheric neutrinos from the decay of short-lived particles in extensive air showers represent the surviving background in cosmic neutrino searches. A conventional component, coming from the decay of pions and kaons, here described by the Honda et al. flux [16], can be modelised as a power law with spectral index $\Gamma$ asymptotically going to 3.7 . The prompt component, expected from charmed hadrons, yields a harder neutrino energy spectrum $(\Gamma \sim 2.7)$. The Enberg et al. [17] prescription is used in this work. Since signal and background spectra are described by power laws with different indices, a cut on the reconstructed energy [18] provides a selection for cosmic neutrinos.

\subsection{Background estimation from data}

A signal from a diffuse emission region in the Southern Sky is searched for by comparing the number of events from the chosen signal region (on-zone) to that coming from regions with no expected signal having the same exposure (off-zones). This choice avoids simulation related biases in the estimation of the signal intensity after event selection. Off-zones are defined as fixed regions in equatorial coordinates which have identical size and shape as the on-zone but have no overlap with it. In local coordinates, off-zones have the same sidereal-day periodicity as the on-zone and span the same fraction of the sky, but with some fixed delay in time. This approach has been already used in ANTARES to search for events from the Fermi bubbles [19]. 9 off-zones are selected for the Galactic plane. Data from the signal region are blinded until the event selection has not been completely defined on Monte Carlo simulations, while data from off-zones can be used to estimate the agreement between data and Monte Carlo, with the expected fluxes introduced in the previos section, as well as the relative agreement between data from different off-zones. Good agreement between data and Monte Carlo, as well as between data from different off-zones, is found.

\subsection{Cut optimisation}

The optimisation of the event selection to enhance the possible cosmic signal against the atmospheric background is made on the basis of track quality parameters to reject wrongly reconstructed atmospheric muons and on the energy estimation to select cosmic neutrinos over the atmospheric background. The optimal selection cut, which maximises the sensitivity, is computed on the basis of the Model Rejection Factor (MRF) procedure [20] for the $\Gamma=2.4$. The final selection cut is $\Lambda>-5.0, \beta<0.5^{\circ}$, 


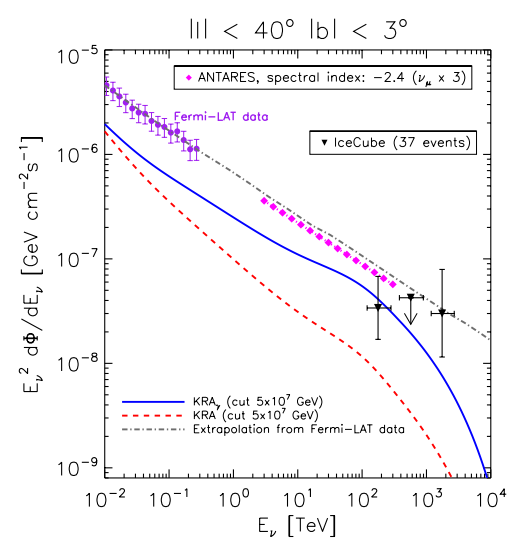

(a)

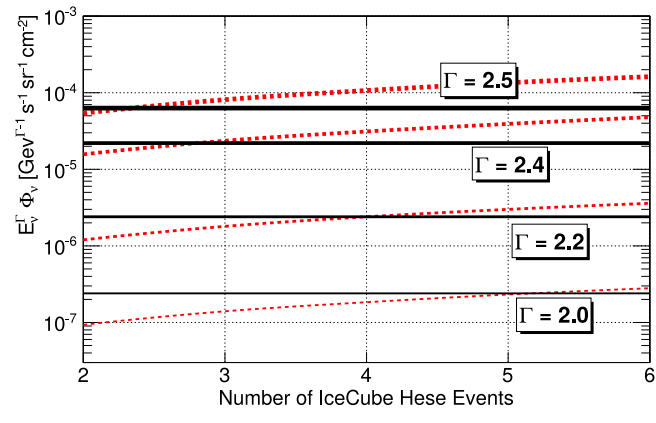

(b)

Figure 1. a) ANTARES upper limit (magenta dots) compared to the expected neutrino fluxes from [12] (blue - solid and red -dashed line for different model assumptions) and the power law extrapolation of Fermi/LAT data from [10] to the IceCube data from that part of the sky. b) ANTARES upper limit for different energy spectra (black solid lines) compared to the cosmic flux (dashed lines) that would produce a certain number of IceCube HESE events from a limited region of the sky.

$\mathrm{E}_{A N N}>10 \mathrm{TeV}$, where $\Lambda$ and $\beta$ are the track quality parameters described in ref. [15], while $\mathrm{E}_{A N N}$ is the energy estimator from the Artificial Neural Network algorithm presented in [18].

\section{Results and outlook}

Considering a signal flux with an energy spectrum $\sim \mathrm{E}^{-2.4}\left(\mathrm{E}^{-2.5}\right)$ the $90 \%$ confidence level sensitivity is 2.0 (6.0) $10^{-5} \mathrm{GeV}^{-1} \mathrm{~cm}^{-2} \mathrm{~s}^{-1} \mathrm{sr}^{-1}$. For comparison, assuming an $\mathrm{E}^{-2.4}$ spectrum, the normalisation of an IceCube-like flux producing 3 or more events from a region in the sky of 0.1 sr is larger than $2.010^{-5} \mathrm{GeV}^{-1} \mathrm{~cm}^{-2} \mathrm{~s}^{-1} \mathrm{sr}^{-1}$ and any of these scenarios can be rejected. Figure $1-$ left, compares the obtained sensitivity in the Galactic Plane region to the expected neutrino flux from CR propagation of [12] and the extrapolated Fermi $\gamma$ spectrum of [10]. This sensitivity holds in the energy range $3 \mathrm{TeV}-$ $300 \mathrm{TeV}$, which contains the central $90 \%$ of the expected signal.

For the Galactic Plane 3.7 events are observed on average for the background regions and two are detected from the on-zone after the final selection. An underfluctuation of the background is thus present in the signal region. Since the Galactic Plane area does not show an excess of events with respect to the background only evaluation, an upper limit at $90 \%$ confidence level on the signal flux can be set and it corresponds to the ANTARES sensitivity for the analysed spectral indices. This upper limit is reported in Fig. 1 right panel, compared to the expected neutrino flux that would induce a certain number of IceCube HESE events. These fluxes have been computed on the basis of the effective areas reported in [2]. Any model producing more than 3 events in the IceCube HESE sample from the Galactic Plane region is excluded at $90 \%$ confidence level for a spectral index larger than 2.4. The upper limits for harder spectral assumptions with the same selection criteria are also shown.

The lack of signal events in ANTARES data significantly constrains the possible Galactic origin of the IceCube Southern sky excess. Adding cascade events in the analysis is foreseen. For these events the angular resolution of ANTARES is about 3 degrees, better than the one of IceCube [21] and the combined search in track and showers can improve the possiblity to observe such a diffuse flux from our Galaxy and to explain the Southern sky excess in IceCube data. 


\section{References}

[1] M. Ageron et al., Nucl. Instrum. Meth. A, 656, 11-38 (2011)

[2] M.G. Aartsen et al., Phys. Rev. Lett., 113, 101101 (2014)

[3] M.G. Aartsen et al., Phys. Rev. Lett., 114, 171102 (2015)

[4] M. Spurio, Phys. Rev. D, 90, 103004 (2014)

[5] M.G. Aartsen et al., Phys. Rev. D, 91, 022001 (2015)

[6] S. Adrián Martínez et al., Astrophys. J. 760: 53 (2012)

[7] J. Barrios Martí, Proceedings of the $34^{\text {th }}$ ICRC, The Hague, Netherlands, ID 1077 (2015)

[8] E. Fermi, Phys. Rev., 75, 1169 (1949); E. Fermi, Astroph. J., 119, 1 (1954)

[9] M. Ackermann et al., ApJ, 750, 3 (2012)

[10] A. Neronov, D. Semikoz, C. Tchernin, Phys. Rev. D, 89, 103002 (2014)

[11] Y.Q. Guo, H.B. Hu \& Z. Tian, arXiv: 1412.8590 (2014)

[12] D. Gaggero et al., ApJ Letters 815: L25 (2015)

[13] A. Neronov \& D. Semikoz, Astropart. Phys. 75: 60-63 (2016)

[14] A. Marinelli et al., Proceedings of the $34^{\text {th }}$ ICRC, The Hague, Netherlands, ID 1126 (2015)

[15] S. Adrián Martínez et al., ApJ Letters, 786, L1 (2014)

[16] M. Honda et al., Phys. Rev. D, 75, 043006 (2007)

[17] R. Enberg et al., Phys. Rev. D, 78, 043005 (2008)

[18] J. Schnabel, Nucl. Instr. and Meth. A, 725, 106-109 (2013)

[19] S. Adrián Martínez et al., Eur. Phys. J. C, 74, 2701 (2014)

[20] G.C. Hill \& K. Rawlins, Astrop. Phys., 19, 393 (2003)

[21] T. Michael, Proceedings of the 34th ICRC, The Hague, Netherlands, ID 1078 (2015) 